\title{
EVOLUTION OF HOMOPHONES AND SYNTACTIC CATEGORIES NOUN AND VERB
}

\author{
Mieko Ogura $^{* 1}$ \& William S-Y. Wang ${ }^{2}$ \\ *Corresponding Author: ogura-m@tsurumi-u.ac.jp \\ ${ }^{1}$ Linguistics Laboratory, Tsurumi University, Yokohama, Japan \\ ${ }^{2}$ Language and Cognitive Sciences, Hong Kong Polytechnic University, Hong \\ Kong, China
}

\begin{abstract}
We examine the neural substrates of comprehension of the bisyllabic homophones in English and Japanese. The evolution of homophones is a result of interaction between the speaker's production and the listener's perception, and the cortical representation of speech does not merely reflect the external acoustic environment. We further demonstrate that noun and verb categories are represented in different neural substrates in English, while both categories are processed in the same anatomical area in Japanese. We suggest that nouns and verbs are originally processed in the same anatomical area of the brain when the basic word order is SOV, and discourse organization is closely interwoven with syntactic organization as shown in Old English and Japanese. When the SOV order is changed to SVO through embedding, a strictly syntactic organization of the clause appears. The noun/verb distinction is clearly visible in the positions that nouns and verbs occupy in sentences, and it is represented in different neural substrates in the brain as shown in Present-day English.
\end{abstract}

\section{Introduction}

Zipf (1949) suggests the simultaneous minimization of the two opposing forces from listener and speaker for form and meaning associations. Zipf's law, which states that word frequencies decay as a power law of its rank, is the outcome of form-meaning associations adopted for complying with listener and speaker needs. Zipf's law implies one form to many meanings, i.e., polysemy and homophony. Polysemy and homophony are the necessary conditions for symbolic systems. Homophony is a desirable feature in communication system and likely results from ubiquitous pressure from efficient communication (Ferrer i Cancho \& Solé 2003, Piantadosi et al. 2012, Wang 2011, Ogura forthcoming).

In this study, we examine the neural substrates of comprehension of homophones. We investigate the evolution of homophones as a result of 
interaction between the speaker's production and the listener's perception. We further demonstrate that noun and verb categories are represented in different neural substrates in English, while both categories are processed in the same anatomical area in Japanese, and explore why such differences arose.

\section{Neural Substrates of Bisyllabic Noun-Verb Homophones in English}

Sereno (1986) conducts the analysis of bisyllabic noun-verb ambiguous pairs, i.e., words with both noun and verb instances. The 570 bisyllabic noun-verb ambiguous words in the Brown Corpus are analyzed in terms of location of the stressed syllable and frequency dominance of the grammatical category. Words are categorized as either forestressed, backstressed, or variably stressed. Dominance is characterized as noun-dominant, verb-dominant, or equidominant. A noun- or verb-dominant word is a word which occurs more than $50 \%$ of the time as a noun or verb, respectively. The vast majority (90\%) of the ambiguous group includes words that maintain a constant stress pattern across syntactic function. They show consistent stress placement depending on their dominant grammatical class usage: Forestressed stimuli are much more likely to be noun-dominant $(75 \%)$ than verb-dominant $(22 \%)$ while backstressed stimuli are only marginally more noun-dominant $(55 \%)$ than verb-dominant $(43 \%)$.

Sereno \& Jongman (1995) investigate systematic acoustic differences in bisyllabic noun-verb ambiguous pairs. 5 speakers read 16 bisyllabic words in both noun and verb contexts. The 16 bisyllabic homophones consist of four categories: 4 pairs which occur more frequently as forestressed nouns (favor, poison, practice, struggle); 4 pairs which occur more frequently as backstressed nouns (control, debate, dispute, report); 4 pairs which occur more frequently as forestressed verbs (handle, notice, rescue, welcome); and 4 pairs which occur more frequently as backstressed verbs (embrace, escape, neglect, reply). They find that stimuli that are more frequent as nouns in English show significantly different first/second syllable amplitude ratios than word stimuli that are more frequent as verbs. In other words, the amplitude of the first syllable of word forms that are usually used as nouns is higher than that of words usually used as verbs, regardless of whether the form in question is being pronounced as a noun or a verb. They conclude that the significant Dominance effects suggest that speakers maximize the difference between noun- and verb-dominant words in conformity with the lexical distribution of English in which the majority of bisyllabic nouns are stressed on the first syllable and the majority of bisyllabic verbs on the second syllable. 
We investigate the neural substrates of bisyllabic noun-verb homophones in English using near-infrared spectroscopy (NIRS). We used 16 bisyllabic noun-verb ambiguous pairs in Sereno \& Jongman (1995). Note the grammatical categories of the words are never ambiguous in the sentence contexts. They are similar in meaning. The stimuli were presented randomly to the 6 adult (both male and female) native English speakers. The subjects were instructed to comprehend the auditorily presented sentences silently. The changes in hemoglobin $(\mathrm{Hb})$ concentrations and their oxygeneration levels in the frontal and temporal lobes in the left hemisphere and the right homologous areas were recorded using NIRS systems (ETG-4000; Hitachi, Tokyo, Japan). The probe pads were positioned onto the subjects' bilateral frontal and temporal

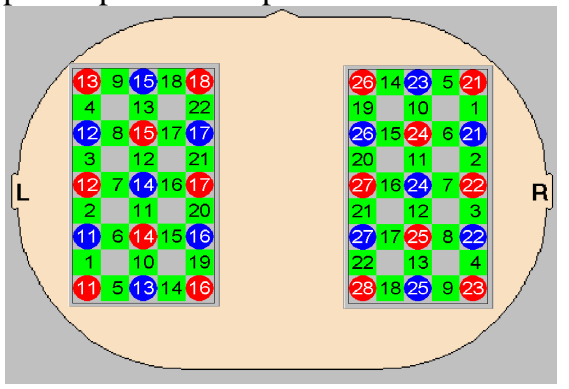
areas as shown in the left figure. Green squares indicate the channel positions. Red probes are emitters and blue ones are detectors. The numbers of the channels $1-22$ correspond to the numbers $1-22$ of the time course of hemoglobin responses in the left and right hemispheres in Figures 1-4.

Figure 1 shows the average values of the oxy-Hb changes of the 6 English subjects in the frontal and temporal lobes in the left hemisphere and the homologous areas in the right hemisphere for the forestressed nouns in solid lines and the forestressed verbs in broken lines in noun-dominant words. We find anatomical-behavioral correlations, with a left frontal cortical network activated for verbs, which is marked with a solid circle, and greater activation in the temporal regions for nouns, which is marked with a dotted circle. In the neuroimaging literature, there is a substantial corpus of studies asking whether nouns and verbs are represented in different neural substrates, or whether both categories are processed in the same anatomical area but with functional differences between them.

Our results show that noun and verb categories are represented in different neural substrates in the left hemisphere. We argue, following Shapiro \& Caramazza (2003), that information about a word's grammatical category is represented independently of its meaning. Semantic information about nouns and verbs is stored in the frontal and temporal lobes, in which categorical distinctions are explicitly represented when nouns and verbs are activated: the 
greater activation of nouns than verbs in the temporal regions and the greater activation of verbs than nouns in the frontal regions. In the right hemisphere the activation of highly frequent nouns in noun-dominant pairs occurs in the frontal and temporal regions. As for the backstressed nouns and backstressed verbs in noun-dominant words, we find little activation of the verbs and the nouns in the frontal and temporal lobes both in the left and right hemispheres.

Table 1 shows the mean, sum of the mean, sum of the square of the mean of oxy-Hb (mM-mm) of the nouns and verbs during the period of $5 \mathrm{~s}$ of a stimulus and $19 \mathrm{~s}$ of the intervals between stimuli at the channels listed in the parentheses by the 6 English subjects in the frontal and temporal lobes of the left hemisphere for the forestressed nouns and verbs in noun-dominant pairs. The degree of freedom and the calculated value of $t$ are given. A value of 2.92 for the frontal lobe and 1.717 for the temporal lobe are needed in a directional test for significance at the $\mathrm{p} \leq 0.05$ level. Since our calculated values are larger than this, we reject the null hypotheses, and conclude that the means of the nouns and verbs do differ both in frontal and temporal lobes.

Table 1. Significant levels in the frontal and temporal lobes for the forestressed noun-dominant pairs.

\begin{tabular}{|c|c|c|c|c|c|c|c|c|c|c|}
\hline \multirow{3}{*}{$\begin{array}{l}\text { noun } \\
\text { verb }\end{array}$} & \multicolumn{5}{|c|}{ frontal area $(13,17)$} & \multicolumn{5}{|c|}{ temporal area $(3,12,21,7,16,11,20,6,15,10,19,14)$} \\
\hline & 0.00065 & 0.0013 & 0.0000335 & \multirow{2}{*}{$\mathrm{df}=2$} & \multirow{2}{*}{$\mathrm{t}=2.97$} & 0.0137916 & 0.1655 & 0.0026962 & \multirow{2}{*}{$\mathrm{df}=22$} & \multirow{2}{*}{$\mathrm{t}=4.59$} \\
\hline & 0.01605 & 0.0321 & 0.0005362 & & & 0.001475 & 0.0177 & 0.0005769 & & \\
\hline
\end{tabular}

Figure 2 shows the verb dominant backstressed nouns and verbs. Verbdominant forestressed nouns and verbs show similar patterns of activation. We find the activation of both forestressed and backstressed verbs in the frontal lobe of the left hemisphere, which is marked with a solid circle. Furthermore, we find the decrease to the negative value in the oxy-Hb for both forestressed and backstressed verbs in the frontal lobe, which is marked with a dotted circle. Task-induced deactivation occurs because certain types of neural processes active during passive states are interrupted when subjects are engaged in effortful tasks (Binder et al. 2009). In the right hemisphere the activation of highly frequent verbs in verb-dominant pairs occurs both in positive and negative values in the frontal regions.

In Table 2 we give the same calculations of the oxy-Hb as in Table 1 for both forestressed and backstressed nouns and verbs in the frontal lobe of the left hemisphere in verb-dominant pairs. A critical value of significance with degree of freedom of 2 at the $\mathrm{p} \leq 0.05$ level is 2.92 , and we conclude that the means of the nouns and verbs do differ. We find the statistically greater activation of verbs than nouns in stressed syllables in the frontal lobes in verb-dominant pairs 


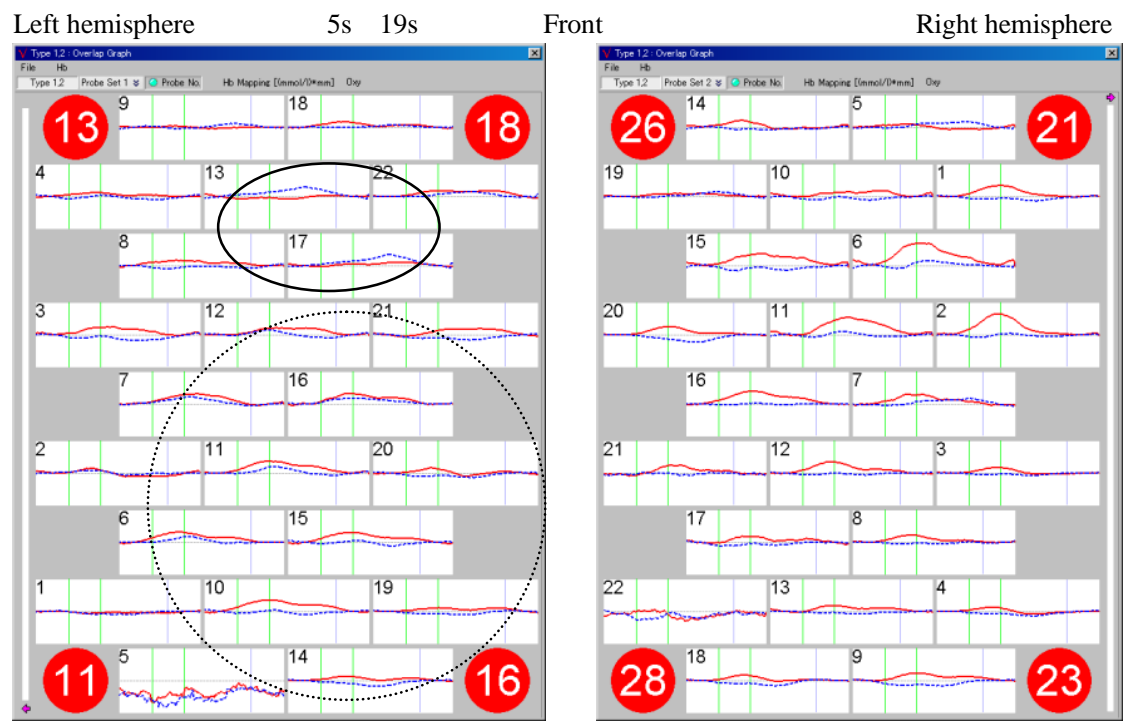

Figure 1 The oxy-Hb changes in the range of 0.10 and $-0.10 \mathrm{mM} \cdot \mathrm{mm}$ at the 22 channels in noundominant forestressed nouns and verbs in English (The sentence is within $5 \mathrm{~s}$, and the interval between sentences is $19 \mathrm{~s}$.)

Left hemisphere

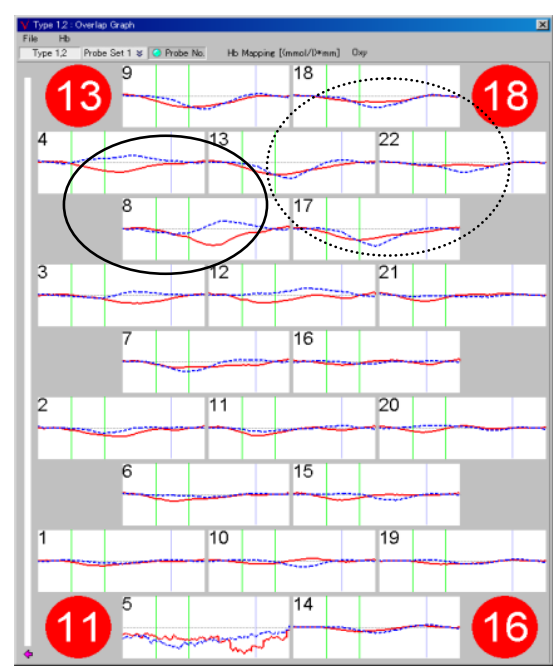

Front

Right hemisphere

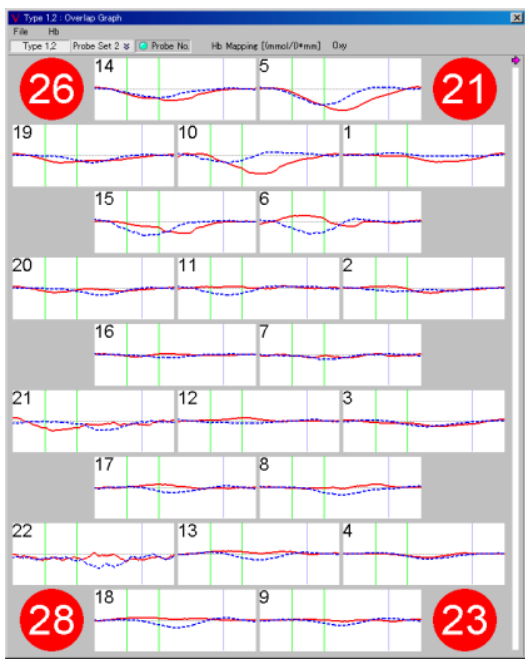

Figure 2 Verb-dominant backstressed nouns and verbs in English

to maximize the difference between noun- and verb-dominance in perception, though the amplitudes in stressed syllables in verb-dominant pairs are lower 
than the amplitude of the first syllable of the forestressed noun-dominant pairs in production. Note that the value of $t$ is only slightly higher than the critical value in the frontal lobe for the forestressed noun-dominant pairs (see Table 1). We suggest that the cortical representation of speech does not merely reflect the external acoustic environment, but instead gives rise to the perceptual aspects relevant for the listener's intended goal.

Table 2. Significant levels in the frontal lobe for the verb-dominant pairs

\begin{tabular}{|c|c|c|c|c|c|c|c|c|c|c|}
\hline \multirow{3}{*}{$\begin{array}{l}\text { noun } \\
\text { verb }\end{array}$} & \multicolumn{5}{|c|}{ frontal area $(4,8)$, forestressed } & \multicolumn{5}{|c|}{ frontal area $(4,8)$, backstressed } \\
\hline & -0.0124 & -0.0248 & 0.000312 & \multirow{2}{*}{$\mathrm{df}=2$} & \multirow{2}{*}{$t=6.00$} & -0.0203 & 0.0406 & 0.0008771 & \multirow{2}{*}{$\mathrm{df}=2$} & \multirow{2}{*}{$\mathrm{t}=3.35$} \\
\hline & 0.02765 & 0.0553 & 0.0016046 & & & 0.00062 & 0.0124 & 0.0001027 & & \\
\hline
\end{tabular}

\section{Neural Substrates of Bisyllabic Homophones in Japanese}

The 8 sentence pairs which contain bisyllabic intra-category noun-noun homophones (e.g. kasa'umbrella', 'bamboo hat') and verb-verb homophones (e.g. $w a k u$ 'boil', 'gush out') were presented auditorily to the 6 adult (both male and female) native Japanese speakers. In Japanese the majority of the homophones are composed of words from the same lexical categories.

Figure 3 shows the average values of the oxy- $\mathrm{Hb}$ changes of the 6 Japanese subjects in the frontal and temporal lobes in the left hemisphere for the first noun in solid lines and the second noun in broken lines of the homophonous noun pairs. We find the activation in middle temporal gyrus which is marked with a solid circle for the first noun, and the activation in inferior frontal gyrus and inferior temporal gyrus which are marked with a dotted circle for the second noun, because there is temporal ambiguity before the noun in Japanese SOV sentences. Figure 4 shows the homophonous verb pairs. The first verb and the second verb show similar patterns of activation. They are not ambiguous in the sentence contexts, because the noun is given before the verbs. Most importantly nouns and verbs are processed in the same anatomical area in the brain in Japanese.

\section{Neural Substrates of Nouns and Verbs}

Hurford (2014) discusses the evolution of nouns and verbs. Noun and verb are the basic major word classes, or syntactic categories. Some languages get close to making no distinction between nouns and verbs, while in other languages the distinction is clearly visible in the positions that words occupy in sentences. In this dimension some languages have more grammar than others. How did this most basic grammatical distinction evolve? Hurford suggests that the answer 


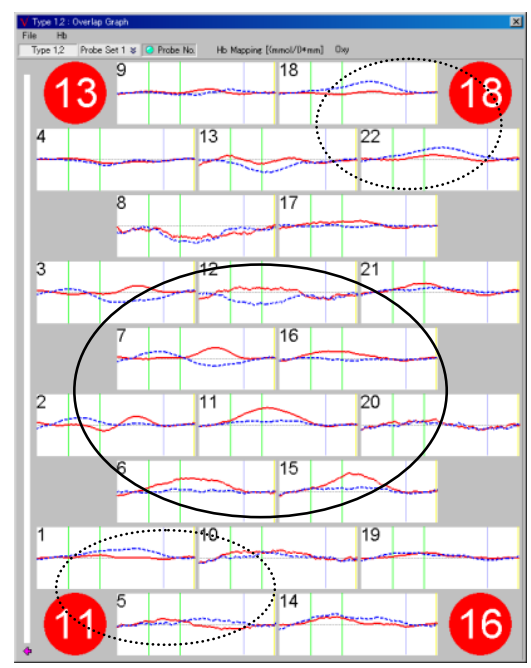

Figure 3 Homophonous noun pairs in Japanese

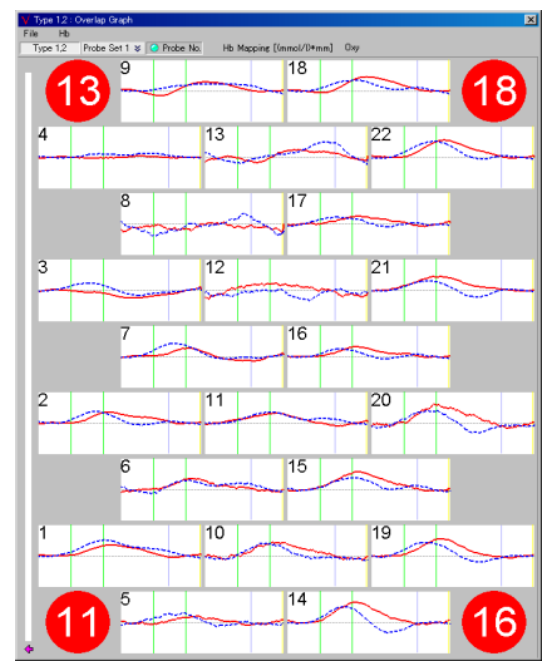

Figure 4 Homophonous verb pairs in Japanese

lies in the central special function of communication in language: giving information about identified objects. In the most ordinary kind of simple sentence, the subject expression with a noun at its core, is the Topic, and the predicate expression, with a verb at its core, is the Comment. This is the original basis for the pervasive noun/verb distinction at the heart of the grammar of languages.

Kemenade \& Los (2006) discuss, based on the syntactic and discourse properties of the adverbs $b a$ and ponne, that Old English (OE) discourse organization was closely interwoven with syntactic organization. The transition of Middle English (ME) is marked by the elimination of the multiple topics in $\mathrm{OE}$, and results in a more strictly syntactic organization of the clause.

We assume that the earliest human language had SOV word order (Newmeyer 2000, Goldin-Meadow et al 2008, Ogura \& Wang 2012), and in OE the basic word order of SOV was intertwined with various types of word order including verb second motivated by discourse organization. Ogura \& Wang $(2012,2014)$ assert that the evolution of the postnominal relative clause in English from independent sentences through the paratactic adjunctive stage to the embedded structures, and its product, the SVO order, brought about a strictly syntactic organization of the clause in ME.

With this transition, the periphrastic constructions of progressive, perfect, pluperfect and modal auxiliaries, definite article and periphrastic $d o$ arose due to the speakers' desire to be more specific and informative than was possible with 
the older forms, and case and gender as concordial categories disappeared. Also we assert that nouns in the Topics and verbs in the Comments which were strongly triggered by the discourse considerations in OE were clearly specified in the positions that they occupy in sentences in ME. We suggest that nouns and verbs were processed in the same anatomical areas of frontal and temporal lobes before $\mathrm{OE}$ as shown in Japanese; after ME semantic information about nouns and verbs is stored in the frontal and temporal lobes, in which categorical distinctions are explicitly represented.

Japanese is SOV language, and nominal relations are expressed by postpositional particles. While the SOV order is basic, Japanese allows reordering of preverbal major constituents. Furthermore, the subject is not expressed in colloquial speech if it is known from the context. We, therefore, find that nouns and verbs are processed in the same anatomical areas of frontal and temporal lobes in Japanese.

\section{Conclusion}

We have investigated the evolution of homophones as a result of interaction between the speaker's production and the listener's perception. In so doing, we have demonstrated that noun and verb categories are represented in different neural substrates in Present-day English. Semantic information about nouns and verbs is stored in the frontal and temporal lobes, in which categorical distinctions are explicitly represented when nouns and verbs are activated. In contrast, both noun and verb categories are processed in the same anatomical area, the frontal and temporal lobes, in Japanese.

We have suggested that nouns and verbs are originally processed in the same anatomical area of the brain when the basic word order is SOV, and discourse organization is closely interwoven with syntactic organization as shown in OE and Japanese. When the SOV order is changed to SVO through embedding, a strictly syntactic organization of the clause appears. The noun/verb distinction is clearly visible in the positions that nouns and verbs occupy in sentences, and it is represented in different neural substrates in the brain as shown in Present-day English.

\section{Acknowledgements}

M. Ogura is supported by the grants from the Human Frontier Science Program and the Ministry of Education, Culture, Sports, Science and Technology of Japan. W. S-Y. Wang is supported by HKRGC-GRF number 14611615. 


\section{References}

Binder, Jeffrey, R. et al. (2009). Where is the semantic system? A critical review and meta-analysis of 120 functional neuroimaging studies. Cerebral Cortex 19, 2767-2796.

Ferrer i Cancho, R. and R. V. Solé (2003) Least effort and the origins of scaling in human language, PNAS 100, 788-791.

Goldin-Meadow, S. et al. (2008). The natural order of events: how speakers of different languages represent events nonverbally". PNAS, 105, 9163-9168.

Hurford, J. R. (2014). The Origins of Language: A slim guide. Oxford: Oxfod University Press.

Kemenade, Ans van \& Bettelou Los (2006). Discourse adverbs and clausal syntax in Old and Middle English. In A. v. Kemenade and B. Los (Eds.),The Handbook of the History of English (pp. 224-248). Oxford: Blackwell.

Newmeyer, F. J. (2000). On the reconstruction of 'proto-world' word order. In C. Knight, M. Studdert-Kennedy and J. R. Hurford (Eds.), The Evolutionary Emergence of Language (pp.372-388). Cambridge: Cambridge University Press.

Ogura, M. (forthcoming). Language Evolution as a Complex Adaptive System: A multidisciplinary approach to the history of English. New York: Oxford University Press.

Ogura, M. \& W. S-Y. Wang (2012). Ambiguity resolution and evolution of word order. In T. C. Scott-Phillips et al. (Eds.), The Evolution of Language, (pp. 274-281). Singapore: World Scientific.

----- (2014). Evolution of tense and aspect. In E. A. Cartmill et al. (Eds.), The Evolution of Language, (pp. 213-220). Singapore: World Scientific.

Piantadosi, S. T. et al. (2012). The Communicative function of ambiguity in language. Cognition 122, 280-291.

Sereno, J. A. \& Jongman, A (1995). Acoustic correlates of grammatical class. Language and Speech 38(1), 57-76.

Shapiro, K. \& A. Caramazza (2003). The representation of grammatical categories in the brain. Trends in Cognitive Sciences 7(5), 201-206.

Wang, W. S-Y. (2011). Ambiguity in language. Korea Journal of Chinese Language and Literature 1, 3-20.

Zipf, K. (1949). Human Behavior and the Principle of Least Effort: An Introduction toHuman Ecology. Cambridge, MA: Addison-Wesley. 